\title{
Experimental and Numerical Study of Mild Steel Behaviour under Cyclic Loading with Variable Strain Ranges
}

\author{
Paulina Krolo, Davor Grandić, and Željko Smolčić \\ Department of Structural Engineering and Technical Mechanics, Faculty of Civil Engineering, University of Rijeka, \\ Radmile Matejčić 3, 51000 Rijeka, Croatia
}

Correspondence should be addressed to Paulina Krolo; paulina.krolo@uniri.hr

Received 30 July 2016; Revised 11 October 2016; Accepted 23 October 2016

Academic Editor: Paolo Ferro

Copyright (C) 2016 Paulina Krolo et al. This is an open access article distributed under the Creative Commons Attribution License, which permits unrestricted use, distribution, and reproduction in any medium, provided the original work is properly cited.

\begin{abstract}
To simulate the effect of variable strains on steel grades S275 and S355, an experimental displacement control test of plate specimens was performed. Specimens were tested under monotonic and cyclic loading according to the standard loading protocol of SAC 2000. During experimental testing, strain values were measured with an extensometer at the tapered part of the specimen. Strains obtained by the experimental tests are disproportional to the applied displacements at the ends of the specimens. This phenomenon occurs due to the imperfections of the specimen, hardening of the material, and the buckling behaviour that appears in real structures due to the high deformation experienced during earthquakes. Due to the relative simplicity and wide applicability of the Chaboche hardening model of steel, the calibration of hardening parameters based on experimental test results was conducted. For the first time, calibration of steel hardening parameters was performed following the Chaboche procedure to define the cyclic behaviour with variable strain ranges. The accuracy of the hardening model with variable strain ranges, which were simulated using ABAQUS software, was verified using the experimental results.
\end{abstract}

\section{Introduction}

Seismic resistant steel structures designed as a dissipative structure must allow for plastic deformation to develop in its specific members [1]. The common practice is to increase the hysteretic energy as much as possible through inelastic behaviour using the ductile properties of the structure. "Plastic" members consisting of mild carbon steel (S235 to S355) in terms of ductility, strength, and stiffness will dissipate seismic energy. Under extreme seismic action, structural steel members, especially the dissipative elements, have to resist enormous cyclic displacement, which is classified as lowcycle fatigue and characterized by repeated inelastic strain leading to material failure. The seismic resistance of the structure is estimated on the basis of structural displacements and preservation of its integrity at the largest displacements that are expected for the earthquake $[2,3]$. The structure displacements in earthquake engineering are expressed as the maximum displacement of structures in the form of interstorey drift and rotation of structural member ends or their connections [4]. The response of these elements mainly depends on the geometric dimensions and hysteretic behaviour of the material [5].

In the structures that are subject to earthquake action, the occurrence of large displacements results in inelastic deformation of the material from which the structure is built. Under cyclic loading, structural steel exhibits complicated mechanical behaviour, which includes the Bauschinger effect as well as hardening behaviour. The effect of loading history on the cyclic behaviour of different types of structural steel is given in [5-7], demonstrating that the responses of structural steel under cyclic and monotonic loading are quite different. With increasing cyclic loops, structural steel exhibits cyclic hardening behaviour, and the hysteresis stress-strain curve is much higher than the monotonic stress-strain curve after steel yielding. The local strain of the material in the plastic deformation areas of structural members and connections of steel frames are not proportional to the displacements. This suggests that varying the symmetric cycle of displacement in the structure leads to unsymmetrical and variable local strain of materials [8]. This occurs due to the local hardening of materials, imperfections in the structural elements, and local 
buckling of the structure [9]. In experimental tests focusing on the connections, the relationship between the stresses and variable strains of materials under cyclic changes of the displacements cannot be determined $[8,9]$, even though it is possible to measure the local strains because the local stresses in the material cannot be measured.

To simulate the effect of variable strains that are not proportional to displacements, an experimental programme is selected in which the test specimens are subjected to uniaxial cyclic loading using displacement control by moving crosshead. A total of 24 specimens of the S275 and S355 steel are tested under the monotonic ( 9 specimens) and cyclic (15 specimens) loading, according to the standard loading protocol of SAC 2000 [10]. During experimental testing, strain values were measured with an extensometer at the tapered part of the specimen. Additionally, the axial force in the specimens and displacements of the moving crosshead were measured and recorded. Based on the measured axial forces, the stresses in the materials at a tapered part of the specimen were determined.

Due to the high cost of these experimental tests, the numerical simulation technique has been widely used, and it is a very powerful tool in the field of structural design. To accurately simulate the behaviour of a given structure, the behaviour of material should be carefully defined in a structural model. Experimental studies provide basic knowledge about the seismic performance of structural steel; however, those experimental results also need to be defined in constitutive form for further implementation in numerical simulation. Most of the calculations are performed using a standard tensile test, which is based on uniaxial loading and the stress-strain relationship, commonly defined as bilinear or multilinear. Such test results are useful only for simple elastic problems or elastoplastic problems with low plastic deformation. However, these models cannot provide an accurate simulation of steel material under cyclic loading. Many researchers have proposed constitutive models to simulate material under cyclic loading. Ramberg and Osgood [11] proposed a three-parameter stress-strain constitutive model of the skeleton curve, which is widely used in metal materials. The application of this model is shown $[7,12]$ on structural steel. Chaboche [13] proposed a cyclic constitutive model that includes the isotropic and kinematic hardening and is used to simulate the inelastic behaviour of materials that are subjected to cyclic loading. This model is applicable in most finite element software in which the nonlinear combined hardening model is supported. Calibration of hardening parameters of materials is usually conducted on specimens exposed to symmetrical cycles, with a constant strain range [14-21]. The resulting parameters are then used in the numerical simulation for solving different engineering problems. However, the structure under the influence of the earthquake action is exposed to variable strain ranges. The complex hardening law of steel is not practical for application due to changes in the strain ranges of the materials that are used in the structure when exposed to an earthquake of random nature. Due to the relative simplicity and wide applicability of the Chaboche hardening model of steel in existing software packages, the calibration of hardening

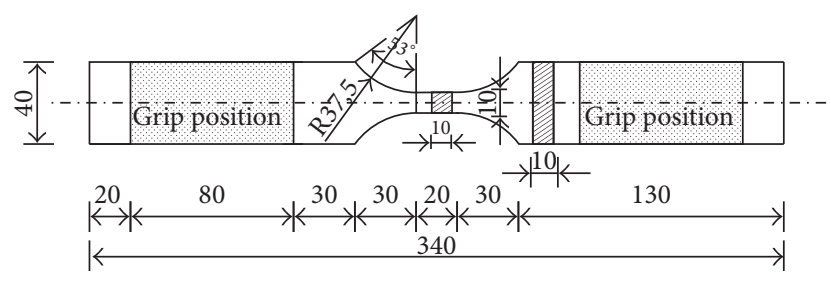

FIGURE 1: Shape and size of the specimen ( $\mathrm{mm})$.

parameters based on experimental test results is conducted. To verify the accuracy of the hardening model with variable strain ranges, experimental results will be simulated using ABAQUS software [22].

\section{Experimental Study}

2.1. Description of Specimens and the Device. As the plated elements are widely used in engineering structures, the plate specimens are adopted in the experimental study instead of test pieces with round cross sections. The materials used in this research were European mild hot-rolled structural steel S275 and S355. A total of 24 plate specimens were tested; the specimen shapes and sizes are shown in Figure 1. The loading device used was the Zwick/Roell Z600, which is designed to perform the tensile and compression tests (see Figure 2). The strains were measured with a gauge length of $20 \mathrm{~mm}$. The minimum yield strength was specified as $275 \mathrm{MPa}$ and $355 \mathrm{MPa}$ for steel grades S275 and S355, respectively, for thicknesses below $16 \mathrm{~mm}$. The steel S275 should exhibit the ultimate tensile strength within the range of $430 \mathrm{MPa}$ and $580 \mathrm{MPa}$ for thicknesses below $16 \mathrm{~mm}$, whereas the steel S355 should exhibit a value within the range of $470 \mathrm{MPa}$ and $630 \mathrm{MPa}$ for thicknesses below $16 \mathrm{~mm}$, according to the EN 10025 standard [23]. The chemical composition of the liquid alloy provided by the processing factory is shown in Table 1 . $\mathrm{M}$ specimens are used for the monotonic tests, whereas $\mathrm{C}$ specimens are used for cyclic tests.

During the experimental tests, in addition to the strain value, the axial force in the specimens and displacements of the moving crosshead were also recorded. Stress is defined as the ratio between the axial force and the initial cross section area at a tapered part of the specimen. Thus, the stress-strain curves for monotonic and cyclic loading (hysteresis curve) were obtained. Test management and registration of the data were conducted using testXpert II software [24].

2.2. Monotonic Test. To verify the mechanical properties of steel grades S275 and S355, the uniaxial tensile tests were performed according to the standard for metallic materials EN ISO 6892-1:2009 [25] for 9 specimens at room temperature. The strains were measured by the extensometer on the gauge length of $20 \mathrm{~mm}$. Test values of Young's modulus $E$, yield stresses $f_{y}$, ultimate stresses $f_{u}$, yield strains $\varepsilon_{y}$, ultimate strains $\varepsilon_{u}$, and fracture strains $\varepsilon_{u 1}$ are summarized in Table 2, while the monotonic loading stress-strain curves are shown in Figure 3. The results of the steel S275 and steel S355 used in this research demonstrate that the yield stress $f_{y}$ and 

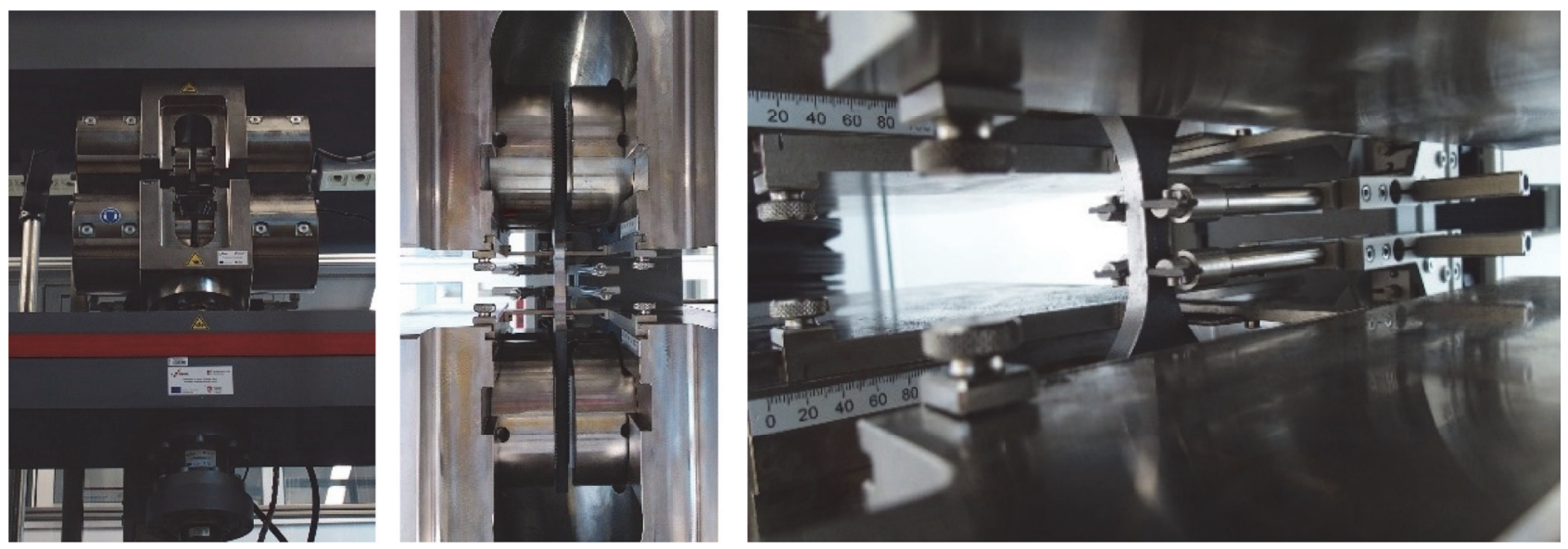

FIgURE 2: Details of the device.

TABLE 1: Chemical composition of liquid alloys of S275 and S355 (\%).

\begin{tabular}{|c|c|c|c|c|c|c|c|c|c|c|c|c|c|c|c|}
\hline & Steel grade & $\mathrm{C}$ & $\mathrm{Mn}$ & $\mathrm{Si}$ & $\mathrm{P}$ & $\mathrm{S}$ & $\mathrm{N}$ & $\mathrm{Cu}$ & $\mathrm{Ni}$ & $\mathrm{Cr}$ & Mo & $\mathrm{V}$ & $\mathrm{Al}$ & $\mathrm{Ti}$ & $\mathrm{Nb}$ \\
\hline \multirow[b]{2}{*}{ EN 10025 standard } & S275 & $<0.18$ & $<1.5$ & $<0.55$ & $<0.03$ & $<0.03$ & $<0.012$ & $<0.55$ & - & - & - & - & - & - & - \\
\hline & S355 & $<0.2$ & $<1.6$ & $\begin{array}{l}>0.14 \\
<0.25\end{array}$ & $<0.03$ & $<0.03$ & $<0.012$ & $<0.55$ & - & - & - & - & - & - & - \\
\hline \multirow{2}{*}{ Measured value } & S275 & 0.173 & 1.02 & 0.021 & 0.016 & 0.005 & 0.0051 & 0.008 & 0.012 & 0.014 & 0.001 & 0.001 & 0.05 & 0.001 & 0.001 \\
\hline & S355 & 0.16 & 1.36 & 0.192 & 0.015 & 0.007 & 0.005 & 0.04 & 0.02 & 0.07 & 0.01 & 0.01 & 0.041 & $<0.001$ & 0.03 \\
\hline
\end{tabular}

TABLE 2: Main mechanical properties of specimens for S275 and S355 obtained by monotonic loading.

\begin{tabular}{|c|c|c|c|c|c|c|}
\hline Type & $E$ [GPa] & $f_{y}[\mathrm{MPa}]$ & $f_{u}[\mathrm{MPa}]$ & $\varepsilon_{y}[\%]$ & $\varepsilon_{u}[\%]$ & $\varepsilon_{u 1}[\%]$ \\
\hline M_S275_01 & 213 & 323 & 471 & 0.187 & 19.04 & 50.2 \\
\hline M_S275_02 & 203 & 309 & 470 & 0.188 & 18.92 & 50 \\
\hline M_S275_03 & 209 & 318 & 471 & 0.175 & 18.93 & 50.1 \\
\hline Mean & 208 & 317 & 471 & 0.183 & 18.96 & 50.1 \\
\hline M_S355_01 & 208 & 385 & 542 & 0.221 & 15.69 & 47.5 \\
\hline M_S355_02 & 195 & 380 & 553 & 0.222 & 15.05 & 47.3 \\
\hline M_S355_03 & 200 & 373 & 546 & 0.244 & 15.17 & 47.2 \\
\hline M_S355_04 & 199 & 399 & 556 & 0.228 & 14.99 & 46.8 \\
\hline M_S355_05 & 205 & 386 & 569 & 0.289 & 15.40 & 47.4 \\
\hline M_S355_06 & 188 & 381 & 548 & 0.243 & 15.32 & 47.4 \\
\hline Mean & 199 & 384 & 552 & 0.288 & 15.27 & 47.3 \\
\hline
\end{tabular}

ultimate stress $f_{u}$ are in the range specified in the standard. The elongation value of steel shown in Table 2 indicates the high ductility of S275 and S355.

2.3. Cyclic Test. The purpose of the cyclic experimental test is to evaluate the seismic performance of material as an important part of the structure. A series of cyclic loading tests were programmed according to the standard loading protocol by SAC 2000 [10] (see Figure 4). The SAC programme was originally developed as a basic loading protocol for the beam to column connections in steel moment resisting frames. The basic loading history is the multiple step test, in which the loading (deformation) history consists of stepwise increasing cycles. The displacement parameter, which is used to control the loading history, is the interstorey drift angle. Since the material is an important component of the structure, the SAC protocol, which simulates the impact of earthquakes, was chosen for an experimental test of steel as a displacement control mode. A total of 15 specimens with grades of S275 and S355 were tested for the same loading protocol and five different maximum strain values (see Table 3). Maximum displacements $\delta_{G}$ are shown in Table 3, corresponding to the strain of steel at which significant strains of members were developed, and seismic energy was dissipated. The 


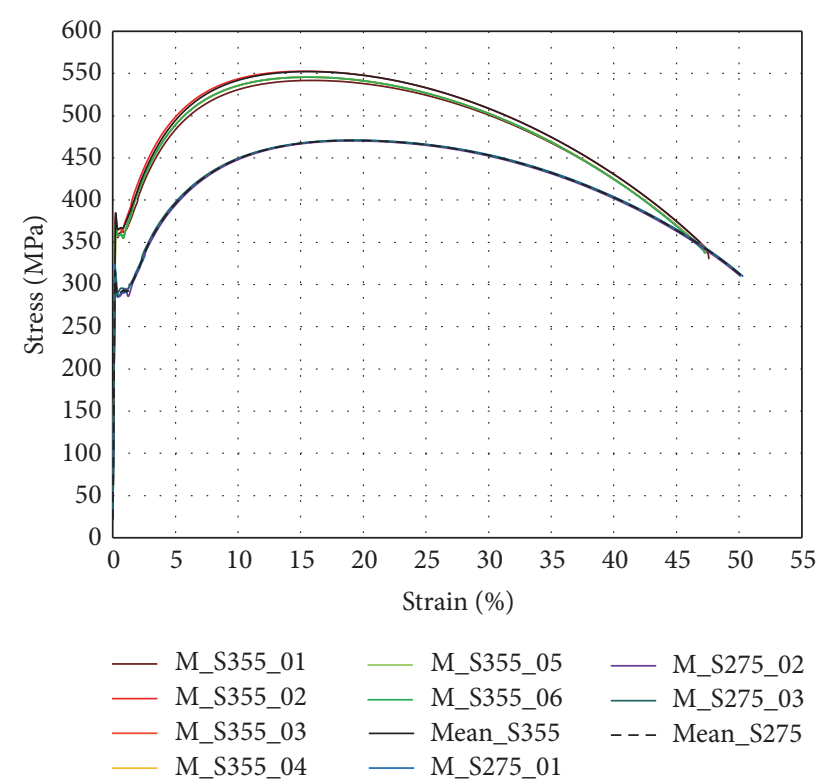

FIGURE 3: Stress-strain curves for monotonic loading.

maximum displacement of $5.23 \mathrm{~mm}$ (Lo. 1), according to the monotonic test (see Figure 4), corresponds to a strain of $15 \%$, the displacement of $3.08 \mathrm{~mm}$ (Lo. 2) corresponds to a strain of $8 \%$, the displacement of $2.02 \mathrm{~mm}$ (Lo. 3) corresponds to a strain of $5 \%$, the displacement of $2.09 \mathrm{~mm}$ (Lo. 4) corresponds to a strain of $5 \%$, and the displacement of $1.73 \mathrm{~mm}$ (Lo. 5) corresponds to a strain of $4 \%$. To reduce the problems of buckling behaviour due to compression strain, the test specimens were shortened, compared to the standard uniaxial tension test specimen. Zhou et al. [5] verified the catted shape of the specimen, and monotonic stress-strain curves obtained from the catted specimen were compared with that using a standard specimen.

The strain was measured using extensometers with a gauge length of $20 \mathrm{~mm}$, and the obtained values were used to control the experimental results. The loading frequency was $0.025 \mathrm{~Hz}(40 \mathrm{~s})$ per each cycle, which includes one tension and one compression half cycle. The first three specimens C_S275_01, C_S275_02, and C_S275_03 were tested under the loading protocol Lo. 1 to maximum displacement values of $5.23 \mathrm{~mm}$ (total of 32 cycles), $4.36 \mathrm{~mm}$ (total of 30 cycles), and $3.42 \mathrm{~mm}$ (total of 28 cycles), respectively. In all three cases, the buckling occurred after 27 cycles, whereas C_S275_01 was fractured in the 32 tensile half cycles. As a result of the testing, strain $\varepsilon$ at time $t$ is obtained and is disproportional to the applied displacements $\delta_{G}$, which are subjected at the ends of the specimen (see Figure 5). This phenomenon occurs due to the imperfections of the specimen and hardening of the material, and buckling occurs in the actual structures due to the large deformation under earthquake action. Specimen C_S275_04 was tested under the loading protocol Lo. 2 to the maximum displacement value of $3.08 \mathrm{~mm}$ (total of 32 cycles), whereas specimens C_S275_05 and C_S275_06 were tested to the maximum strain value of $2.52 \mathrm{~mm}$ (total of 30 cycles). After the last cycle, the specimens were tensile and stretched to fracture (Figure 5).
TABLE 3: Loading protocol details.

\begin{tabular}{lcccccc}
\hline \multirow{2}{*}{ Load step Number of cycles } & \multicolumn{5}{c}{$\delta_{G}[\mathrm{~mm}]$} \\
& & Lo. 1 & Lo. 2 & Lo. 3 & Lo. 4 & Lo. 5 \\
\hline 1 & 6 & 0.60 & 0.45 & 0.40 & 0.47 & 0.46 \\
2 & 6 & 0.67 & 0.50 & 0.42 & 0.51 & 0.48 \\
3 & 6 & 0.94 & 0.59 & 0.49 & 0.56 & 0.53 \\
4 & 4 & 1.25 & 0.70 & 0.54 & 0.63 & 0.57 \\
5 & 2 & 1.82 & 1.00 & 0.67 & 0.78 & 0.68 \\
6 & 2 & 2.38 & 1.33 & 0.85 & 0.97 & 0.82 \\
7 & 2 & 3.42 & 1.94 & 1.25 & 1.35 & 1.12 \\
8 & 2 & 4.36 & 2.52 & 1.64 & 1.73 & 1.43 \\
9 & 2 & 5.23 & 3.08 & 2.02 & 2.09 & 1.73 \\
\hline
\end{tabular}

Capacity reduction due to buckling behaviour occurs only in the specimen C_S275_04. Specimens C_S275_07, C_S275_08, and C_S278_09 were tested under the loading protocol Lo. 3 to the maximum displacement value of $2.02 \mathrm{~mm}$ (total of 32 cycles). No capacity reduction occurred due to the buckling behaviour. Specimens C_S355_01, C_S355_02, and C_S355_03 were tested under the loading protocol Lo. 4 to the maximum displacement value of $2.09 \mathrm{~mm}$ (total of 32 cycles; Figure 5), whereas specimens C_S355_04, C_S355_05, and C_S355_06 were tested under loading protocol Lo. 5 to the maximum displacement value of $1.73 \mathrm{~mm}$ (total of 32 cycles; Figure 5). After the last cycle, specimen C_S355_01 to 06 was tensile and stretched to fracture.

The stress $\sigma$ and strain $\varepsilon$ relationships present the hysteresis curve shown in Figure 6, obtained for both steel grades using five different loading protocols with variable strain ranges.

A comparison between the monotonic stress-strain curve and hysteresis stress-strain curve obtained by cyclic loading is shown in Figure 7. A monotonic stress-strain curve is often used as a constitutive model of the material in seismic calculations. However, hysteresis and monotonic constitutive stress-strain curves are quite different under cyclic loading. Damage accumulation due to cyclic loading leads to ductility reduction, as we can see in the strain results for ultimate strength and fracture strength. The ultimate strength value was almost equal for the monotonic and cyclic loading case. The strain at ultimate strength $\varepsilon_{u}$ and strain at fracture strength $\varepsilon_{u 1}$ for the monotonic and cyclic curve indicate a significant difference. With increasing cyclic loops, the structural steel exhibits cyclic hardening behaviour, and the hysteresis stress-strain curve is much higher than the monotonic stress-strain curve after steel yielding. The fracture strain of steel S275 is maximally reduced by $43.47 \%$, and S355 is reduced by $27.84 \%$ after cyclic loading.

\section{Numerical Study of Cyclic Behaviour}

To simulate the accurate behaviour of materials, the model of material should be carefully defined in the finite element model. Under cyclic loading, with increasing cyclic loops, the steel exhibits hardening behaviour. By repeating the cycle, the 

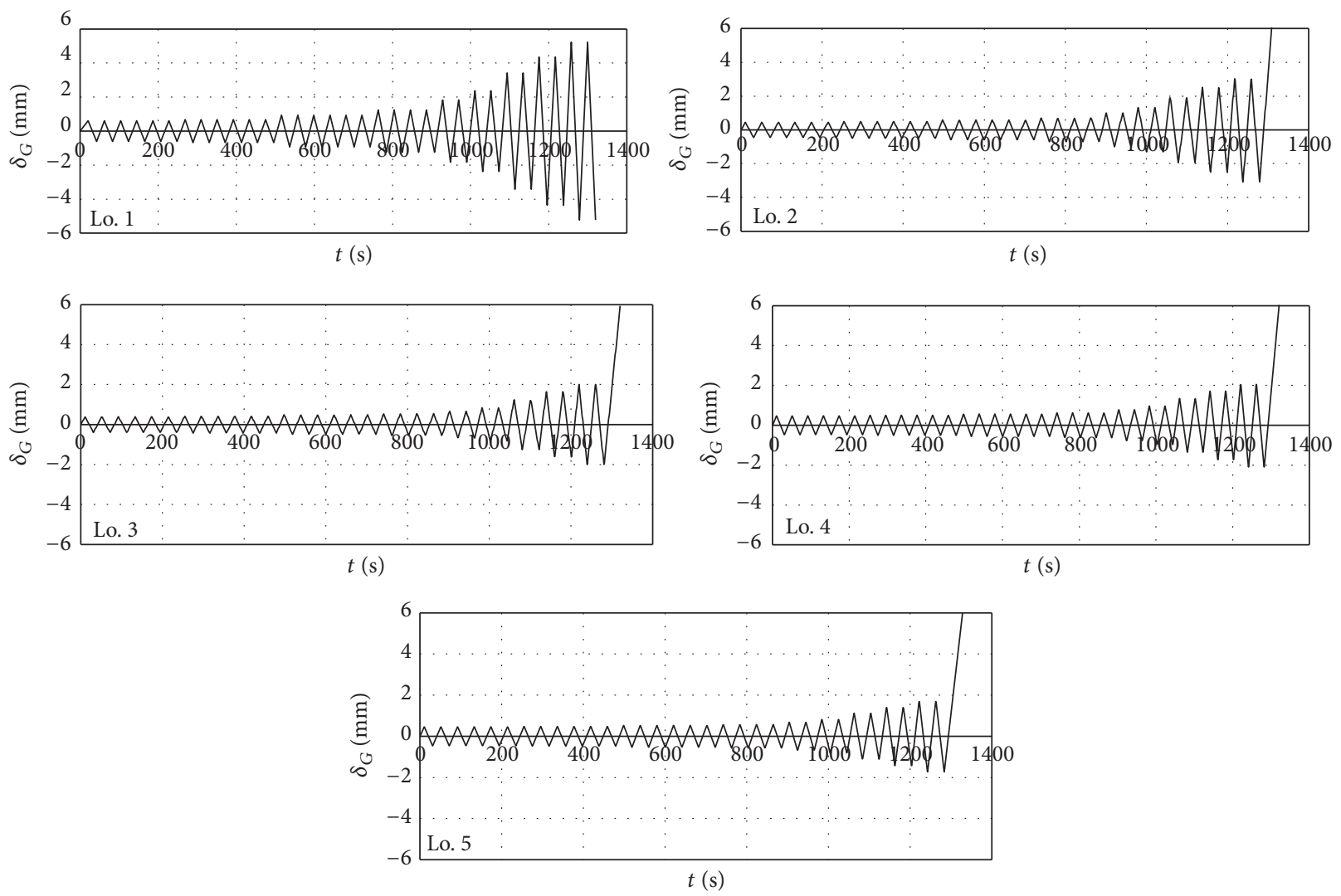

Figure 4: Cyclic loading histories according to SAC 2000 [10].

steel includes the Bauschinger effect, which is characterized by reduced yield stress when the direction of strain is changed and decreases with continued cyclic loading. The accurate constitutive model of steel under cyclic loading is quite important to the numerical simulation. Due to the relative simplicity and wide applicability of the Chaboche hardening model [13] of steel, in most software packages, calibration of hardening parameters based on experimental test results is performed. The accuracy of the hardening model with variable strain ranges was verified using the experimental results that were simulated using ABAQUS software [22]. The nonlinear isotropic/kinematic hardening model was used in the simulation for the von Mises flow rule. The Chaboche hardening model consists of two components: an isotropic hardening component in which the yield surface remains the same shape but expands with increasing stress and a kinematic hardening component in which the yield surface remains in the same shape and size but is translated in the stress space. The cyclic hardening behaviour of steel is described by the isotropic hardening law, whereas the Bauschinger effect is described by the kinematic hardening law.

3.1. Constitutive Model. The yield surface is defined by the function, using the von Mises yield criterion:

$$
f=J_{2}(\sigma-\alpha)-\sigma^{0}=0,
$$

where $\sigma^{0}$ is the yield stress and $J_{2}(\sigma-\alpha)$ is the equivalent von Mises stress with respect to the backstress $\alpha$, defined as

$$
J_{2}(\sigma-\alpha)=\sqrt{\frac{3}{2}\left(S-\alpha^{\mathrm{dev}}\right):\left(S-\alpha^{\mathrm{dev}}\right)},
$$

where $\sigma$ is the stress tensor, $S$ is the deviatoric stress tensor, and $\alpha^{\mathrm{dev}}$ is the deviatoric part of the backstress tensor.

The isotropic hardening behaviour defines the evolution of the yield surface size $\sigma^{0}$ as a function of the equivalent plastic strain $\bar{\varepsilon}^{\mathrm{pl}}$ and can be expressed as the simple exponential law:

$$
\sigma^{0}=\left.\sigma\right|_{0}+Q_{\infty}\left(1-e^{-b \vec{\varepsilon}^{p^{l}}}\right)
$$

where $\left.\sigma\right|_{0}$ is the initial yield stress at zero plastic strain and $Q_{\infty}$ and $b$ are material parameters. $Q_{\infty}$ is the maximum change in the size of the yield surface, and $b$ defines the rate at which the size of the yield surface changes as plastic strain develops.

The kinematic hardening behaviour is defined as an additive combination of a purely kinematic term (linear Ziegler hardening law) and relaxation term, which introduces the nonlinearity and can be expressed as follows:

$$
d \alpha_{i}=\frac{C}{\sigma^{0}}(\sigma-\alpha) d \bar{\varepsilon}^{\mathrm{pl}}-\gamma \alpha_{i} d \bar{\varepsilon}^{\mathrm{pl}},
$$


6

Advances in Materials Science and Engineering

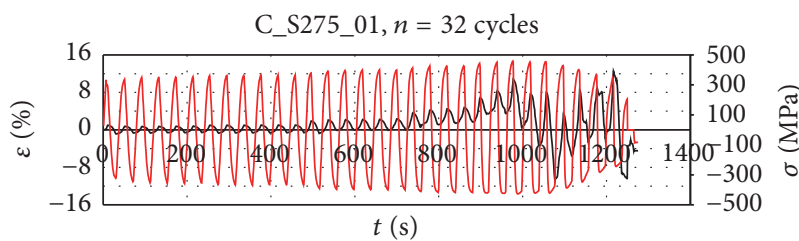

- Strain

- Stress

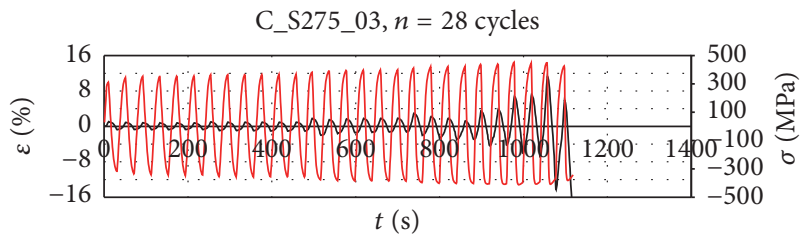

- Strain

- Stress

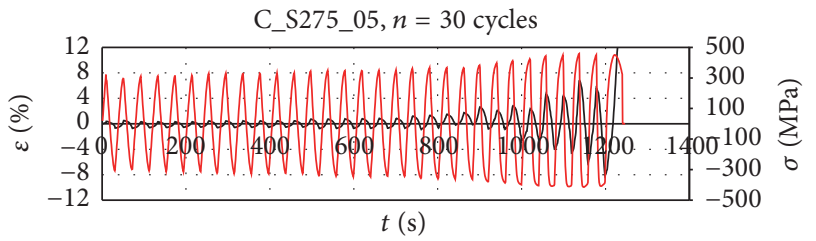

- Strain

— Stress

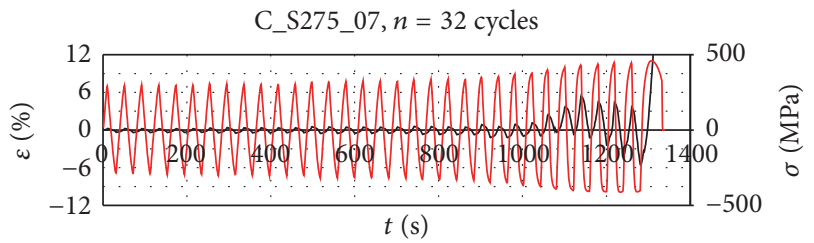

- Strain

— Stress

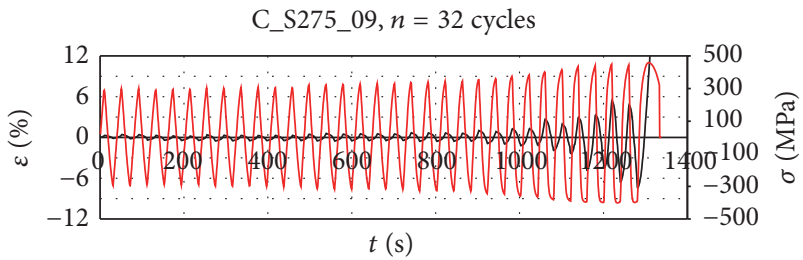

- Strain

- Stress

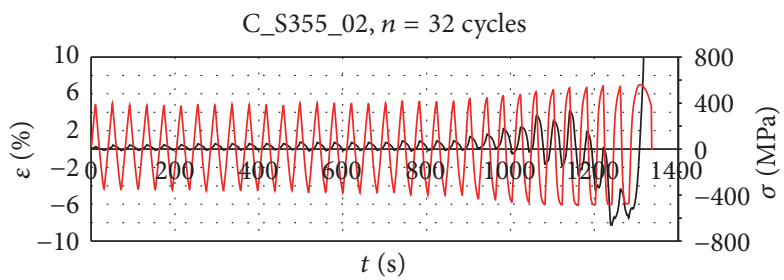

- Strain

- Stress

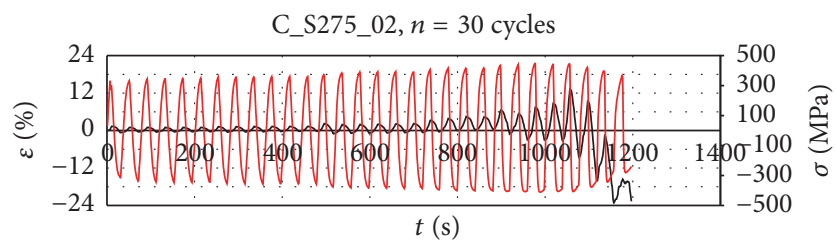

- Strain

_ Stress

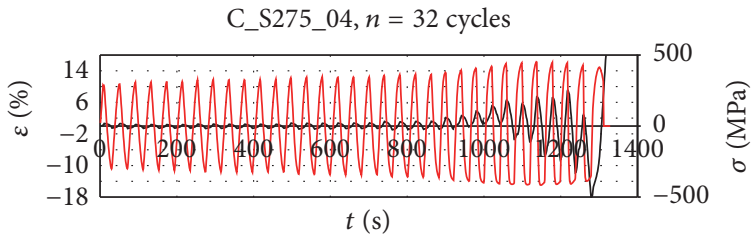

- Strain

— Stress

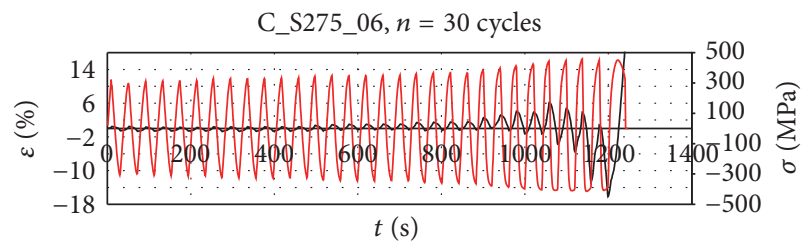

- Strain

- Stress

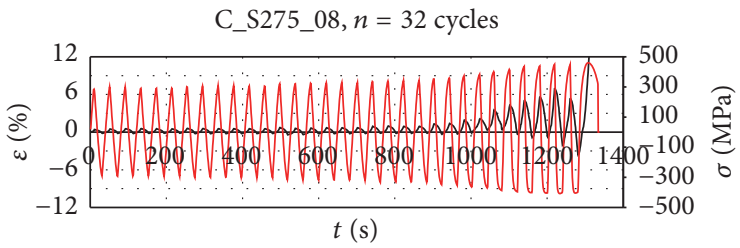

- Strain

- Stress

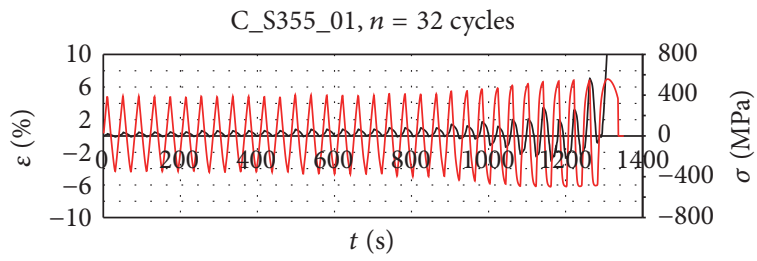

- Strain

- Stress

C_S355_03, $n=32$ cycles

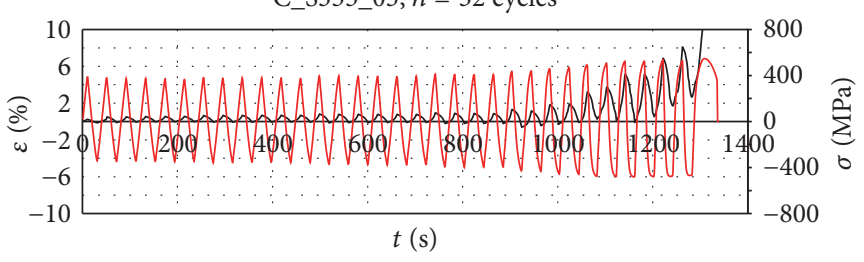

_ Strain

_ Stress

Figure 5: Continued. 

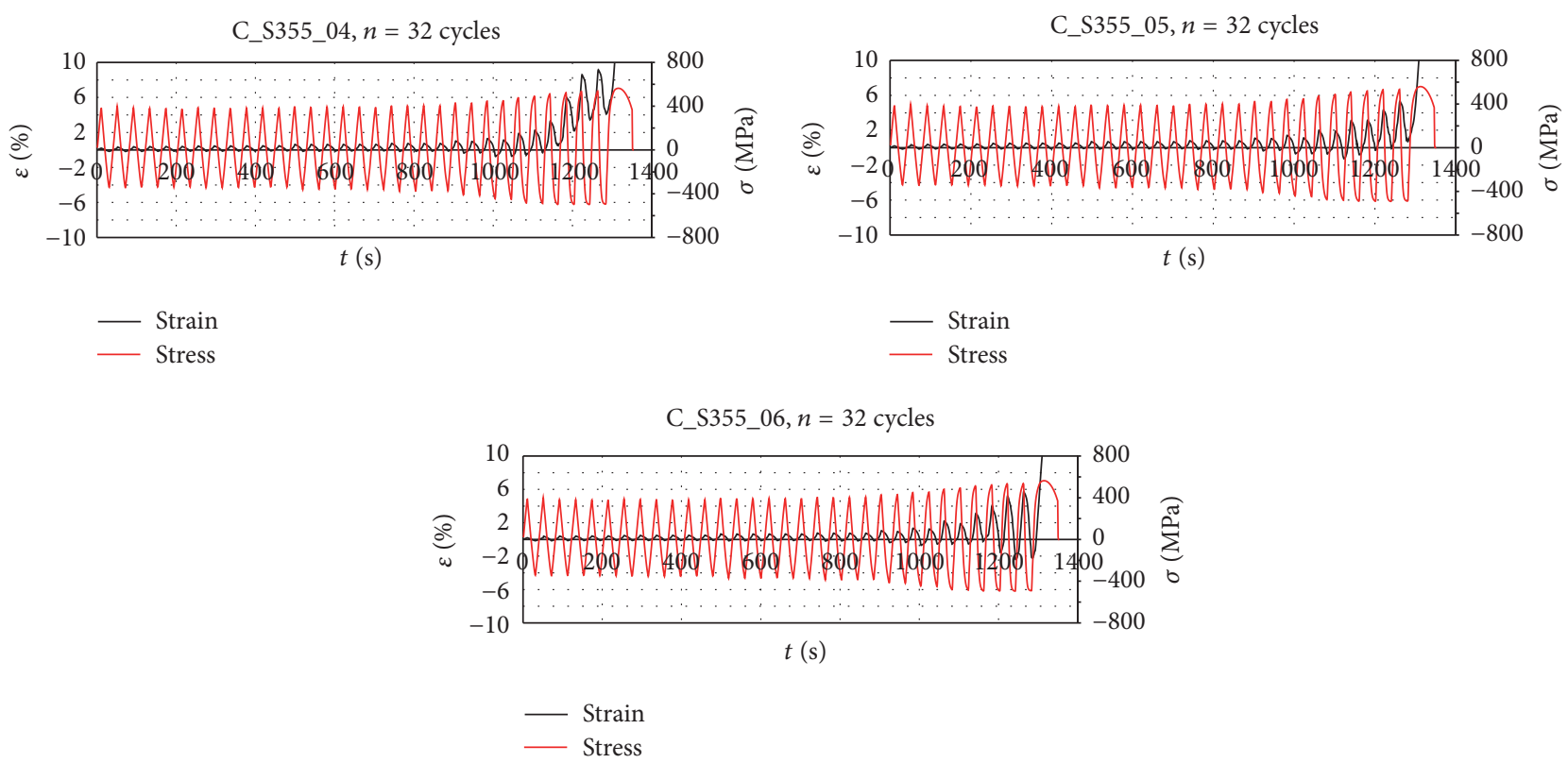

FIGURE 5: Strain and stress records for 15 specimens of steel grades S275 and S355.

where $C$ and $\gamma$ are the material parameters that are calibrated from cyclic test data. $C$ is the initial kinematic hardening module, whereas $\gamma$ determines the rate at which the kinematic hardening module decreases with increasing plastic deformation.

The overall backstress is computed from the following expression:

$$
\alpha=\sum_{i=1}^{N} \alpha_{i},
$$

where $N$ is the number of backstresses; Chaboche proposed using three backstresses $(N=3)$.

3.2. Calibration of the Material Parameters. For the first time, the Chaboche model [13] was used for calibration of isotropic and kinematic hardening parameters $\left(Q_{\infty}, b, C\right.$, and $\gamma$ ) of steel subjected to cyclic behaviour with variable strain ranges. The experimental test results of specimens C_S275_09 and C_S355_06 were used for calibration of isotropic and kinematic hardening parameters. Those two specimens show the most stabilized cycles and minimum effect of buckling behaviour of steel grades S275 and S355, respectively. The numerical model for simulation of the cyclic behaviour of steel is modelled as a unit beam element. That unit beam finite element model represents the tapered part of the tested specimen in which the strains were measured. Parameters $E$ (Young's modulus), $\nu$ (Poisson's ratio), and $\left.\sigma\right|_{0}$ (initial yield stress at zero plastic strain), which describe the elastic behaviour of steel, were defined as the mean values from the experimental tests. The hardening parameters for each steel grade are identified by comparison of the numerically simulated hysteresis stress-strain curve and experimental hysteresis stress-strain curve using the "trial and error method." Strain values $\varepsilon$, which were obtained from the experimental test of specimens C_S275_09 and C_S355_06 (Figure 5), were used as loading protocol in the numerical simulation. The last half cycle of the experimental stabilized hysteresis stress-strain curve is used for calibration of the kinematic hardening parameters $\left(C_{1}, C_{2}, C_{3}, \gamma_{1}, \gamma_{2}, \gamma_{3}\right)$. Figure 8 shows the comparison between the obtained numerical simulations and experimental test of cyclic behaviour for both calibrated specimens. The numerical model does not contain the damage model. The described model shows the behaviour of steel up to the strain value, which corresponds to the ultimate strength obtained by the experimental tests. Calibrated isotropic and kinematic hardening parameters for the steel grades S275 and S355 using previously described protocol and test results of specimens C_S275_09 and C_S355_06 are shown in Table 4.

Numerical simulations performed using the calibrated isotropic and kinematic hardening parameters from Table 4 were also conducted for the other 13 strain protocols (Figure 5) that were not used in the parameter calibration procedure. For example, the numerical simulations of steel specimen C_S275_01 were subjected to the C_S275_01 protocol with 32 cycles of strain amplitudes, as shown in Figure 5, and specimen C_S275_02 was subjected to the C_S275_02 protocol with 30 cycles of strain amplitudes, also shown in Figure 5. The results and discussion of the material behaviour obtained by conducting the numerical simulations are presented in the next chapter.

\section{Results and Discussion}

The comparison between the experimental test and numerical simulation for steel S275 and steel S355 is presented in Figures 9 and 10. Table 5 summarizes the mechanical properties of the experimental test and numerical simulation results for both grades of steel. Table 5 includes the values 

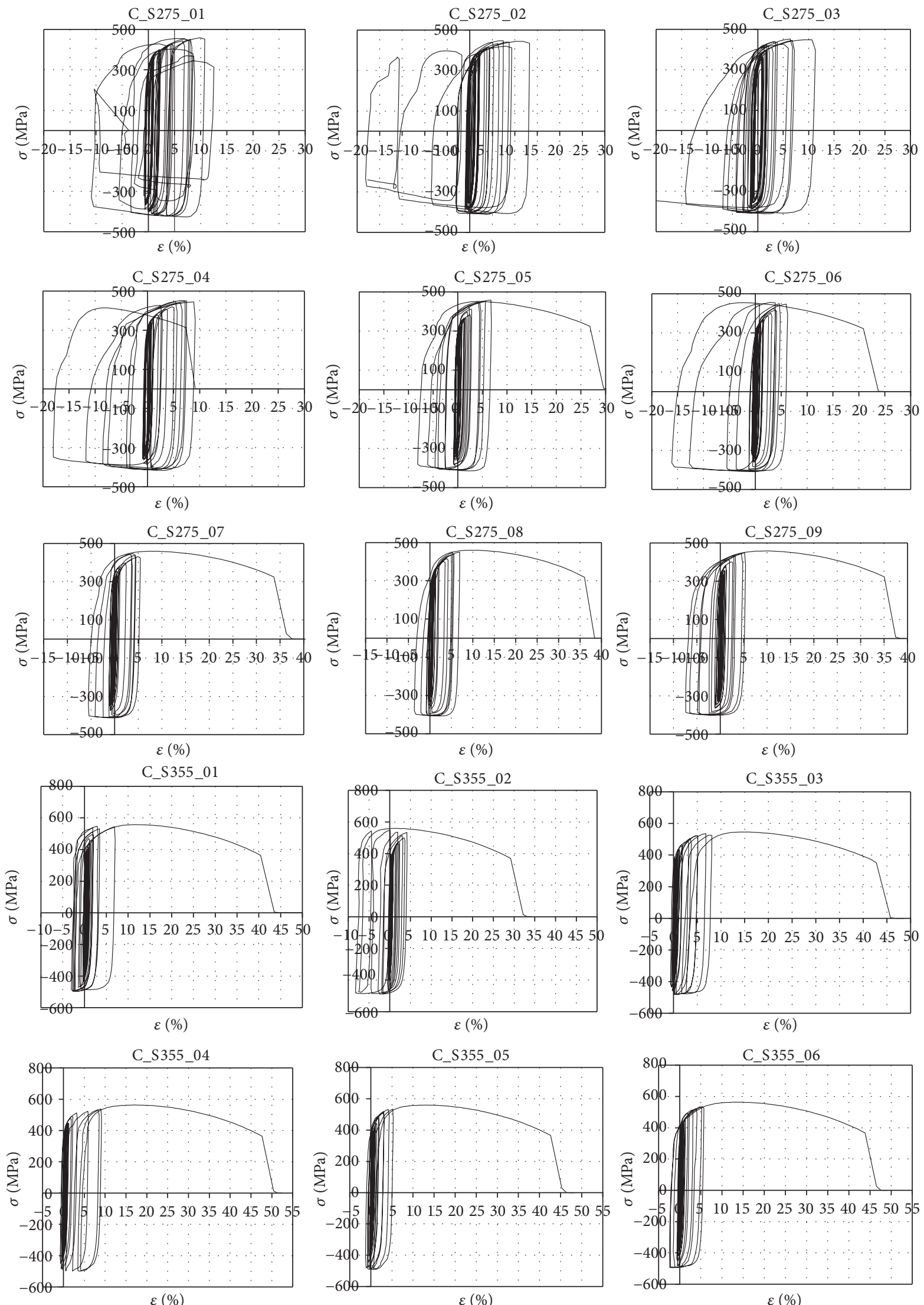

FIgURE 6: The hysteresis stress-strain curves for steel grades S275 and S355. 


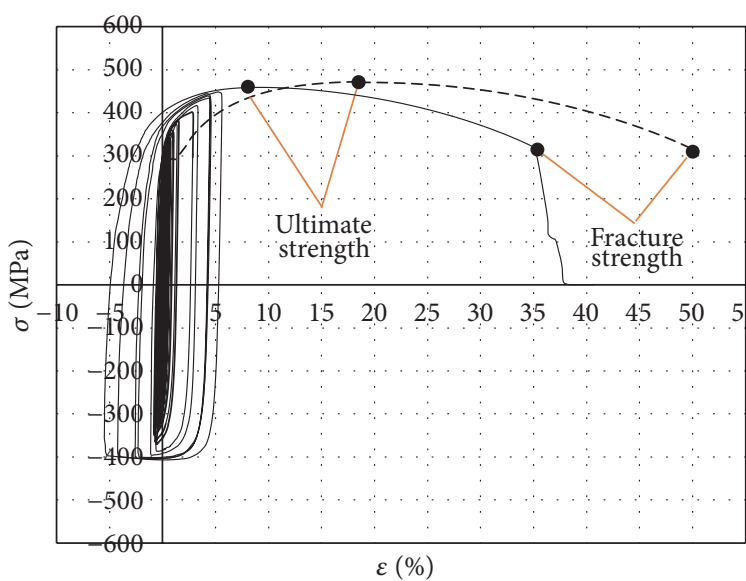

- Mean_cyclic_S275

--- Mean_monotonic_S275

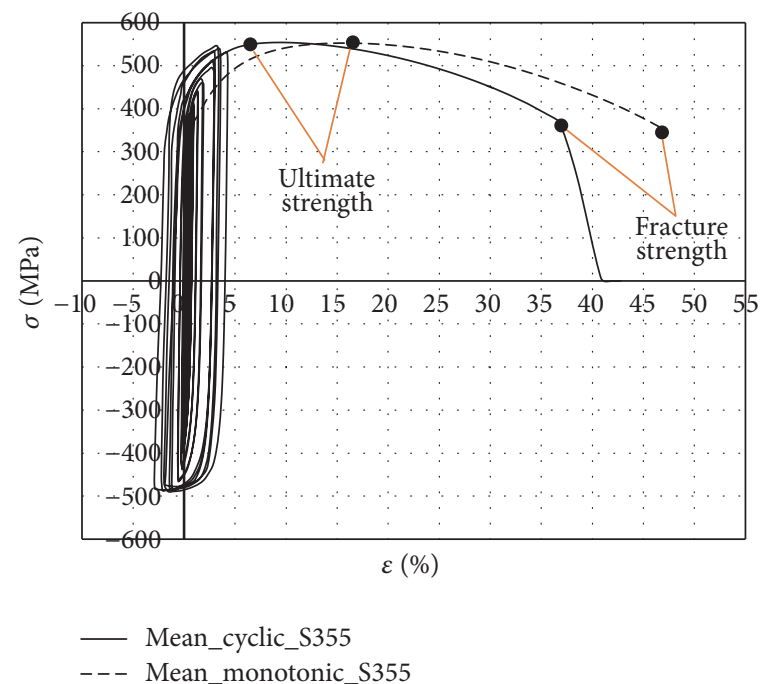

FIGURE 7: Comparison between monotonic loading stress-strain curves and hysteresis stress-strain curves.
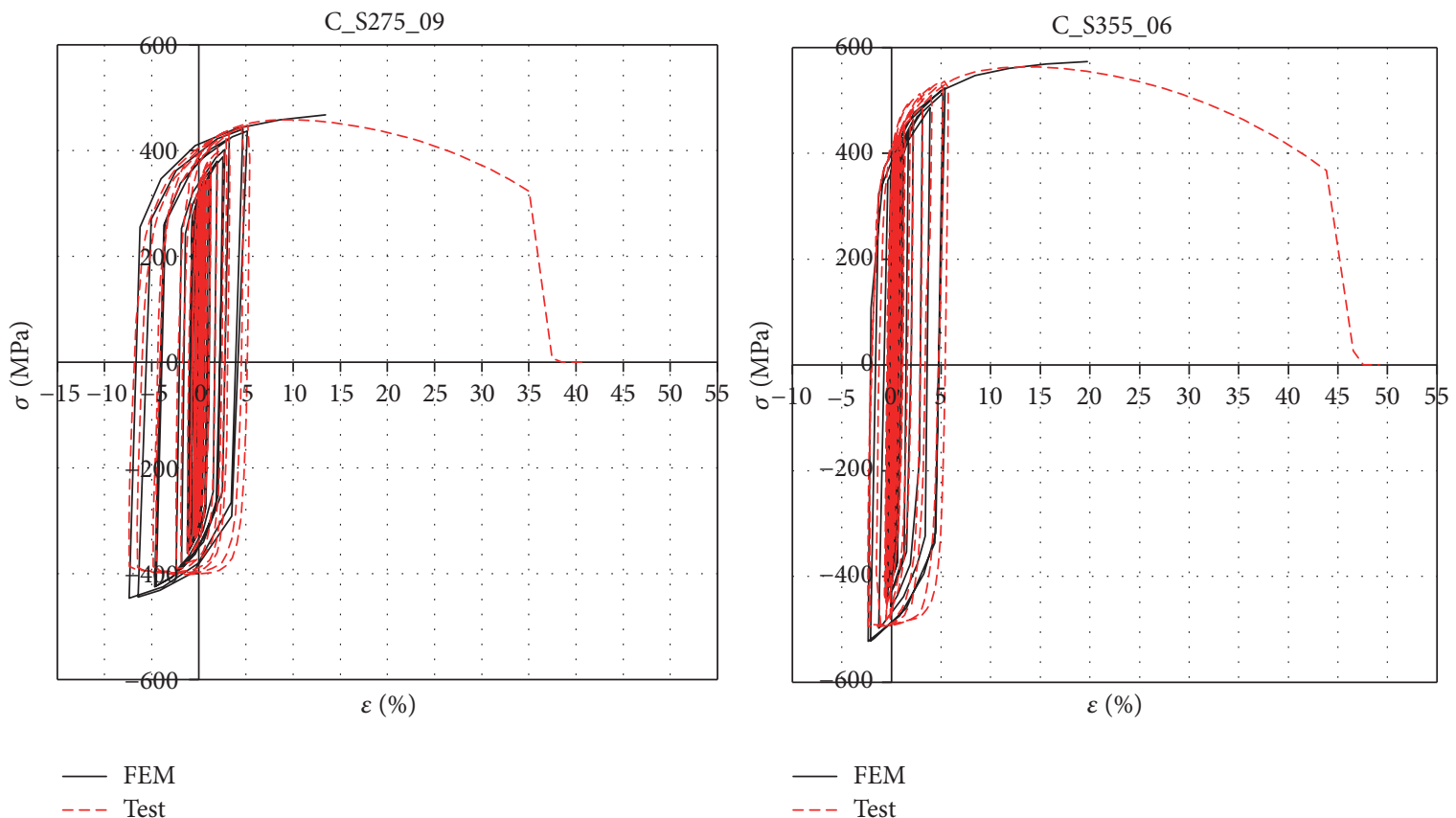

FIgURE 8: Comparison of the numerical simulation and experimental test results for the calibrated specimens.

of Young's modulus $E$, yield stress $f_{y}$, ultimate stress $f_{u}$, yield strain $\varepsilon_{u}$, fracture strain $\varepsilon_{u 1}$, and $\Delta f_{u}$, which represents the absolute deviation of the ultimate stress value obtained numerically in relation to the ultimate stress value obtained by the experimental test. Deviation is calculated according to the following equation:

$$
\Delta f_{u}=\left|\left(\frac{f_{u}(\mathrm{FEM})}{f_{u}(\mathrm{Test})}-1\right) \cdot 100\right| .
$$

The numerical model does not contain the damage model; hence, the fracture strains were obtained only from experimental test data. Hysteresis stress-strain curves obtained by numerical simulation (FEM) fit the hysteresis stress-strain curves obtained by the experimental test (Test) in cycles in which no buckling behaviour was observed (see Figures 9 and 10). Numerical simulations show the visible difference in describing the last few cycles in compression when the buckling occurred. In these cycles, the satisfactory agreement between numerical and experimental results is achieved in tension. The largest buckling behaviour of the tested specimens occurred in the first six tested specimens (C_S275_01 to C_S275_06), which were subjected to loading protocols with the highest level of strain (13.3\% to $-23.2 \%)$. Significantly less buckling behaviour occurred in the tested 

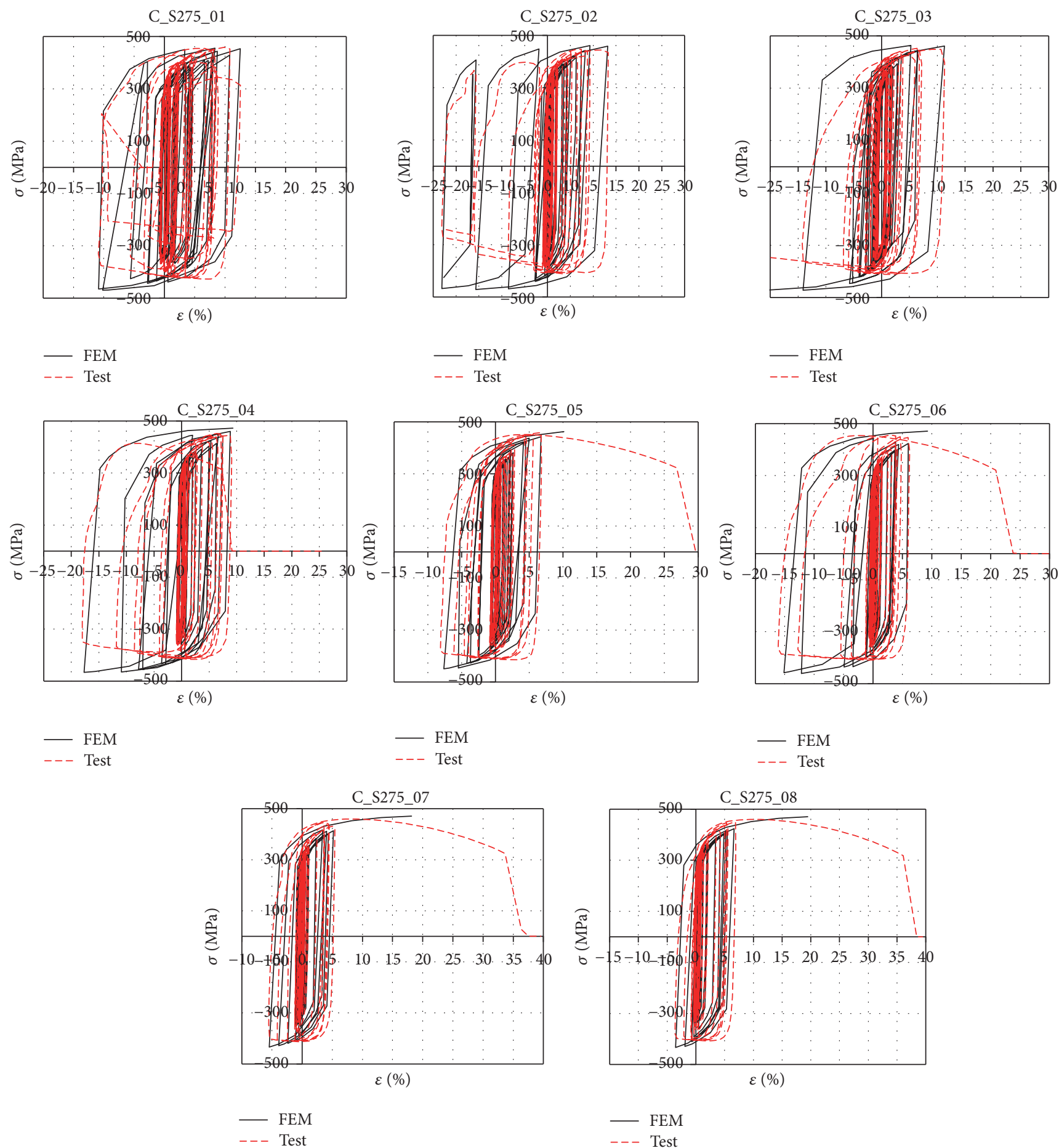

FIGURE 9: Comparison of the numerical simulation and experimental test for steel S275.

specimens C_S275_07 to C_S275_09, which were subjected to a loading protocol with strain levels in the range of $6.9 \%$ to $-6.5 \%$ and C_S355_01 to C_S355_06 with strain levels of $9.2 \%$ to $-7.5 \%$. The buckling problems in the conducted experimental tests occur for all tested specimens only in the last few cycles at each level of strain greater than $2 \%$ for steel grade S275 and 2.8\% for steel grade S355, respectively.
Ultimate stresses obtained by numerical simulation were determined for the strain value that corresponds to the ultimate stresses obtained by the experimental test. As can be observed from Table 5, the differences between the ultimate stresses obtained by the numerical simulation and experimental test for specimens of steel grade S275 show values between $0.44 \%$ and $3.49 \%$, with a mean value of $0.86 \%$. 

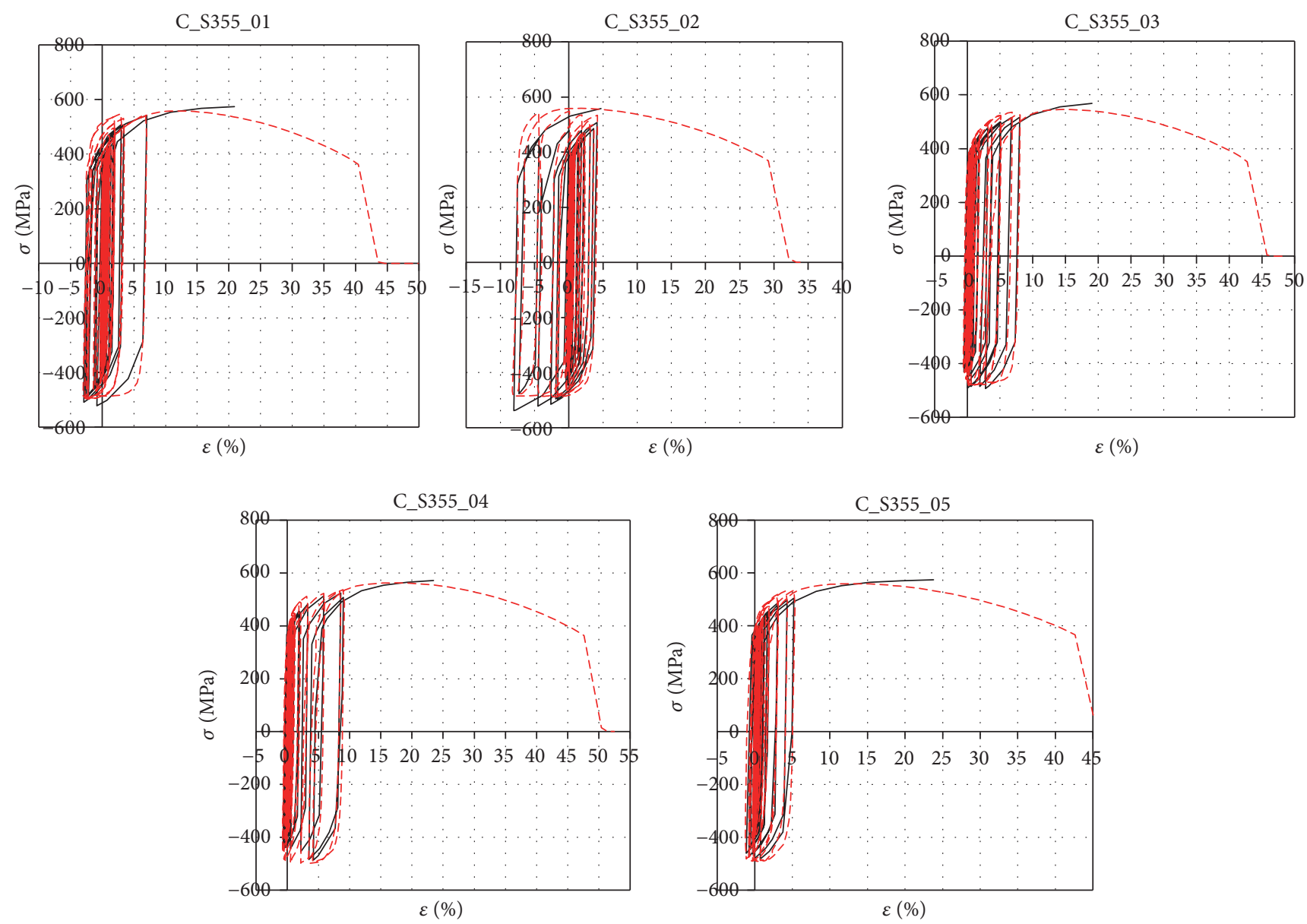

FIGURE 10: Comparison of the numerical simulation and experimental test results for steel S355.

The differences of the ultimate stresses between numerical simulation and experimental test of steel grade S355 take values between $0.18 \%$ and $1.46 \%$, with a mean value of $0.36 \%$.

Based on previous analysis, it can be concluded that the determined hardening parameters obtained by the Chaboche model effectively describe the behaviour of steel grades S275 and S355 under the influence of cyclic loading with variable strain ranges.

\section{Conclusions}

A series of experimental tests were conducted on structural mild carbon steel of S275 and S355 under monotonic and cyclic loading. To simulate real seismic action on the material, a loading protocol was used in experimental testing with variable strain ranges that were not proportional to the applied displacements. During the experimental testing, strain on the specimen was measured with an extensometer. The strain values obtained from the experimental test were then used as loading protocol for a numerical simulation. An accurate constitutive model of steel under cyclic loading is quite important for numerical simulation. For this purpose, the Chaboche hardening model was used for the calibration of the hardening parameters based on the experimental test data. The fundamental contribution of this research is a new approach to the calibration procedure in which the experimental values of strain are used for numerical simulation of steel behaviour. Calibration of hardening parameters of steel according to the Chaboche procedure was applied for the first time to define the cyclic behaviour of steel with variable strain ranges. The hardening model includes isotropic and kinematic hardening. The accuracy of the hardening model with variable strain ranges was verified using the experimental results that were simulated in the ABAQUS software. The analysis includes the stress-strain response, hardening behaviour, and damage evaluation. Based on this work, the following conclusions can be drawn:

(1) The behaviour of S275 steel and S355 steel under cyclic loading and monotonic loading is quite different. Hysteresis stress-strain curves lie much higher than monotonic stress-strain curves between the steel yielding and ultimate strength of cyclically loaded specimens.

(2) Increasing the cyclic loops and strain amplitudes directly affects the material ductility. Ultimate strain and fracture strain are reduced if the cyclic and monotonic stress-strain curves are compared. 
TABLE 4: Calibrated parameters of mild steel grade S275 and S355.

\begin{tabular}{lccccccccccc}
\hline \multirow{3}{*}{ Material } & \multicolumn{3}{c}{ Elastic behaviour } & \multicolumn{4}{c}{ Plastic behaviour } \\
& $E(\mathrm{MPa})$ & $v$ & $\left.\sigma\right|_{0}(\mathrm{MPa})$ & $C_{1}(\mathrm{MPa})$ & $\gamma_{1}$ & $C_{2}(\mathrm{MPa})$ & $\gamma_{2}$ & $C_{3}(\mathrm{MPa})$ & $\gamma_{3}$ & $Q_{\infty}(\mathrm{MPa})$ & $b$ \\
\hline S275 & 207000 & 0.3 & 285 & 13921 & 765 & 4240 & 52 & 1573 & 14 & 25.6 & 4.4 \\
S355 & 185000 & 0.3 & 386 & 5327 & 75 & 1725 & 16 & 1120 & 10 & 20.8 & 3.2 \\
\hline
\end{tabular}

TABLE 5: Summary of experimental and numerical test results of steel specimens of grade S275 and S355.

\begin{tabular}{lcccccccc}
\hline \multirow{2}{*}{ Type } & \multicolumn{3}{c}{$E[\mathrm{GPa}]$} & $f_{y}[\mathrm{MPa}]$ & \multicolumn{2}{c}{$f_{u}[\mathrm{MPa}]$} & \multicolumn{3}{c}{$\Delta f_{u}[\%]$} & $\varepsilon_{u}[\%]$ & $\varepsilon_{u 1}[\%]$ \\
& Test & Test & Test & FEM & & Test & Test \\
\hline C_S275_01 & 206 & 334 & 459 & 454 & 1.09 & 9.88 & - \\
C_S275_02 & 187 & 332 & 447 & 449 & 0.45 & 6.40 & - \\
C_S275_03 & 195 & 279 & 453 & 447 & 1.32 & 6.38 & - \\
C_S275_04 & 199 & 287 & 452 & 449 & 0.66 & 6.38 & - \\
C_S275_05 & 195 & 322 & 459 & 443 & 3.49 & 6.73 & - \\
C_S275_06 & 180 & 287 & 456 & 458 & 0.44 & 2.86 & - \\
C_S275_07 & 218 & 289 & 459 & 453 & 1.31 & 8.10 & 33.67 \\
C_S275_08 & 199 & 282 & 460 & 464 & 0.86 & 10.40 & 36.05 \\
C_S275_09 & 207 & 284 & 458 & 460 & 0.44 & 9.41 & 35.03 \\
C_S355_01 & 191 & 380 & 558 & 555 & 0.54 & 11.64 & 40.43 \\
C_S355_02 & 190 & 384 & 559 & 558 & 0.18 & 3.61 & 29.20 \\
C_S355_03 & 193 & 386 & 546 & 554 & 1.46 & 14.91 & 42.80 \\
C_S355_04 & 192 & 379 & 563 & 560 & 0.53 & 17.61 & 47.62 \\
C_S355_05 & 179 & 382 & 560 & 559 & 0.18 & 12.92 & 42.64 \\
C_S355_06 & 185 & 386 & 563 & 564 & 0.18 & 13.97 & 43.82 \\
\hline
\end{tabular}

(3) The loading history has a significant effect on the cyclic behaviour of steel; the cyclic stress-strain curves are not unique for the same material due to variable strain.

(4) Numerical stress-strain curves using Chaboche hardening parameters agree well with the stress-strain curves obtained by the experimental test.

(5) The obtained hardening parameters can be used in engineering practice and finite element analysis of the cyclic behaviour of steel structures in seismic situations.

(6) The numerical model of steel in this study does not consider the damage model; hence, the model does not account for the decrease in strength after reaching the ultimate strength. This will be analysed in future work.

\section{Competing Interests}

The authors declare that there are no competing interests regarding the publication of this paper.

\section{Acknowledgments}

This work has been supported in part by Ministry of Science, Education and Sports of the Republic of Croatia under the project Research Infrastructure for Campus-based Laboratories at the University of Rijeka (no. RC.2.2.06-0001). The project has been cofunded by the European Fund for Regional Development (ERDF). This research was performed within the scientific project Grant no. 13.05.1.1.01, supported by the University of Rijeka.

\section{References}

[1] D. Grecea, N. Muntean, and D. Dubina, "Control of bolted beam-to-column connections in moment joints by T-stub properties," in Proceedings of the 7th International Conference on Behaviour of Steel Structures in Seismic Areas, pp. 249-254, CRC Press, Santiago, Chile, January 2012.

[2] EN, "Eurocode 8: design of structures for earthquake resistance-Part 1: general rules, seismic actions and rules for buildings," EN 1998-1, European Committee for Standardization (CEN), Brussels, Belgium, 2004.

[3] FEMA, "Improvement of nonlinear static seismic analysis procedure," FEMA 440, Prepared by the Applied Technology Council (ATC-55 Project), Washington, DC, USA, 2005.

[4] V. Gioncu and F. M. Mazzolani, Ductility of Seismic Resistant Steel Structures, Spon Press, London, UK, 2002.

[5] F. Zhou, Y. Chen, and Q. Wu, "Dependence of the cyclic response of structural steel on loading history under large inelastic strains," Journal of Constructional Steel Research, vol. 104, pp. 64-73, 2015.

[6] Y. Shi, M. Wang, and Y. Wang, "Experimental and constitutive model study of structural steel under cyclic loading," Journal of Constructional Steel Research, vol. 67, no. 8, pp. 1185-1197, 2011.

[7] G. Shi, M. Wang, Y. Bai, F. Wang, Y. Shi, and Y. Wang, "Experimental and modeling study of high-strength structural steel under cyclic loading," Engineering Structures, vol. 37, pp. $1-13,2012$.

[8] C.-M. Uang, Q.-S. Yu Kent, S. Noel, and J. Gross, "Cyclic testing of steel moment connections rehabilitated with RBS or welded haunch," Journal of Structural Engineering, vol. 126, no. 1, pp. 57$68,2000$.

[9] G. Shi, Y. Shi, and Y. Wang, "Behaviour of end-plate moment connections under earthquake loading," Engineering Structures, vol. 29, no. 5, pp. 703-716, 2007.

[10] SAC-SteelProject, Protocol for Fabrication, Inspection, Testing and Documentation of Beam-Column Connection Tests and Other Experimental Specimens, FEMA, Oakland, Calif, USA, 1997. 
[11] W. Ramberg and W. R. Osgood, "Description of stress-strain curves by three-parameters," Tech. Rep. 902, National Advisory for Aeronautics, 1943.

[12] P. Dusicka, A. M. Itani, and I. G. Buckle, "Cyclic response of plate steels under large inelastic strains," Journal of Constructional Steel Research, vol. 63, no. 2, pp. 156-164, 2007.

[13] J. L. Chaboche, "Time-independent constitutive theories for cyclic plasticity," International Journal of Plasticity, vol. 2, no. 2, pp. 149-188, 1986.

[14] K. M. Zhao and J. K. Lee, "Finite element analysis of the threepoint bending of sheet metals," Journal of Materials Processing Technology, vol. 122, no. 1, pp. 6-11, 2002.

[15] S. M. H. Kabir, T.-I. Yeo, and S.-H. Kim, "Characterization of material parameters," in Proceedings of the World Congress on Engineering (WCE '09), vol. 2, London, UK, July 2009.

[16] M. Rezaiee-Pajand and S. Sinaie, "On the calibration of the Chaboche hardening model and a modified hardening rule for uniaxial ratcheting prediction," International Journal of Solids and Structures, vol. 46, no. 16, pp. 3009-3017, 2009.

[17] S. J. Zakavi, M. Zehsaz, and M. R. Eslami, "The ratchetting behavior of pressurized plain pipework subjected to cyclic bending moment with the combined hardening model," Nuclear Engineering and Design, vol. 240, no. 4, pp. 726-737, 2010.

[18] H. Wang, Y. Yan, M. Wan, and X. Wu, "Experimental investigation and constitutive modeling for the hardening behavior of 57540 aluminum alloy sheet under two-stage loading," International Journal of Solids and Structures, vol. 49, no. 26, pp. 3693-3710, 2012.

[19] A. Kalnins, J. Rudolph, and A. Willuweit, "Using the nonlinear kinematic hardening material model of Chaboche for elasticplastic ratcheting analysis," Journal of Pressure Vessel Technology, Transactions of the ASME, vol. 137, no. 3, Article ID 031006, 2015.

[20] V. N. Van Do, C.-H. Lee, and K.-H. Chang, "A constitutive model for uniaxial/multiaxial ratcheting behavior of a duplex stainless steel," Materials and Design, vol. 65, pp. 1161-1171, 2015.

[21] M. Zehsaz, F. V. Tahami, and H. Akhani, "Experimental determination of material parameters using stabilized cycle tests to predict thermal ratchetting," UPB Scientific Bulletin, Series D: Mechanical Engineering, vol. 78, no. 2, pp. 17-30, 2016.

[22] ABAQUS, Analysis User'S Manual I_V, Version 6.12, Dassault Systemes, Fremont, Calif, USA, 2012.

[23] C. European Standard, EN 10025: Hot Rolled Products of Structural Steel, European Committee for Standardization, Brussels, Belgium, 2004.

[24] TestXpertII, Instruction Manual, Version 2.2, Zwick GmbH\&Co. KG, Ulm, Germany, 2015.

[25] C. European Standard, EN ISO 6892-1: Metallic MaterialsTensile Testing-Part1: Method of Test at Room Temperature, European Committee for Standardization, Brussels, Belgium, 2010. 

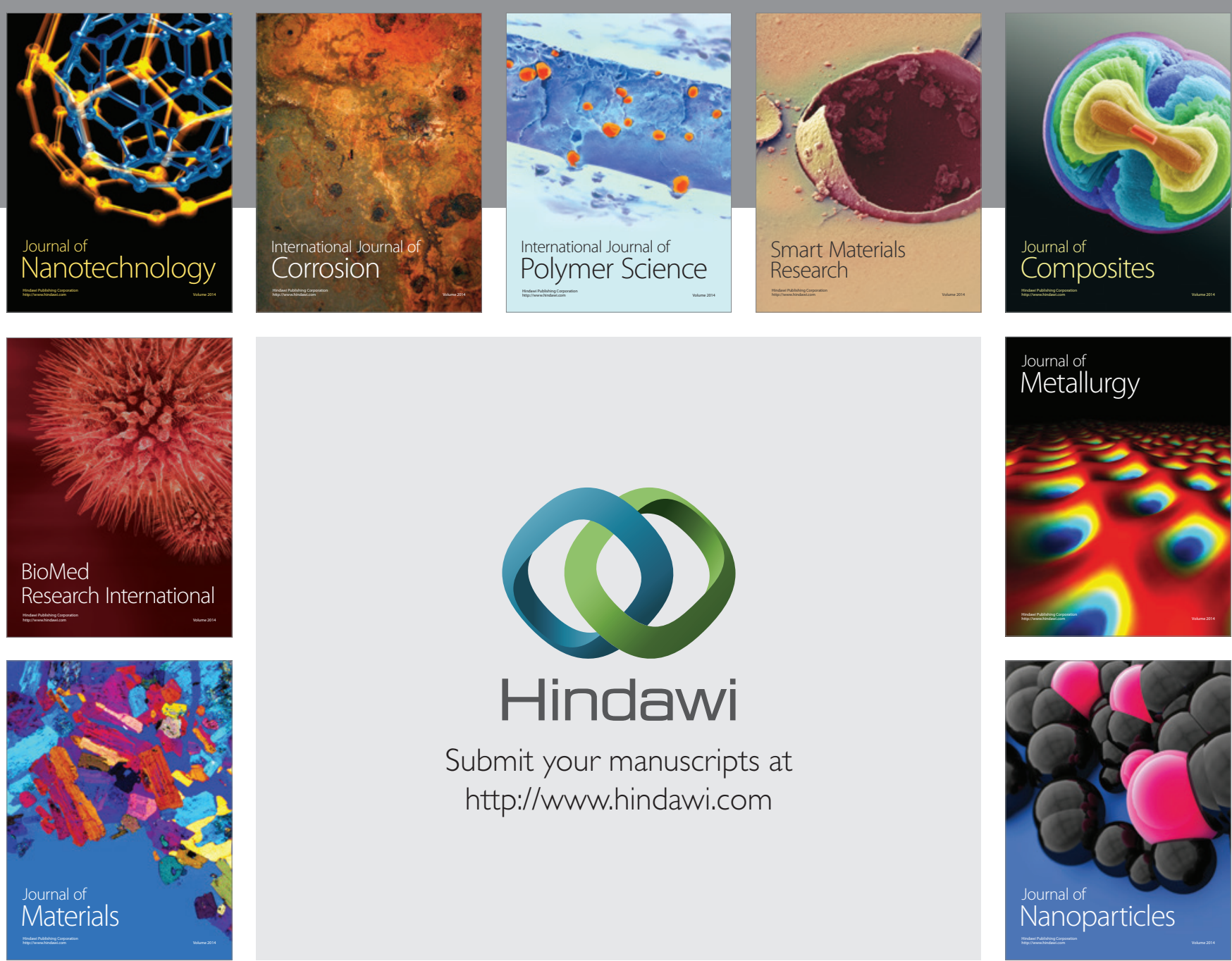

\section{Hindawi}

Submit your manuscripts at

http://www.hindawi.com

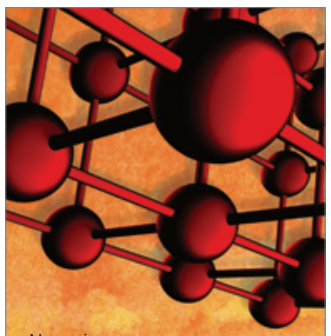

Materials Science and Engineering
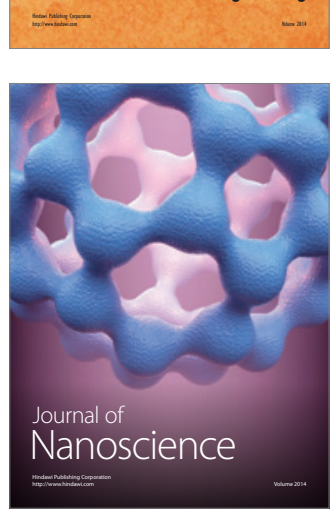
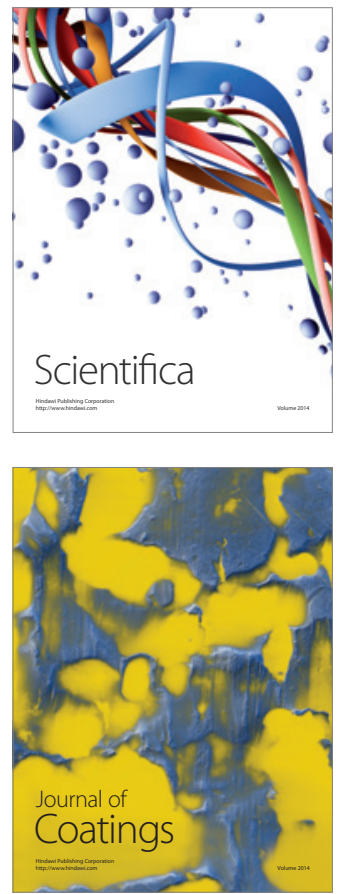
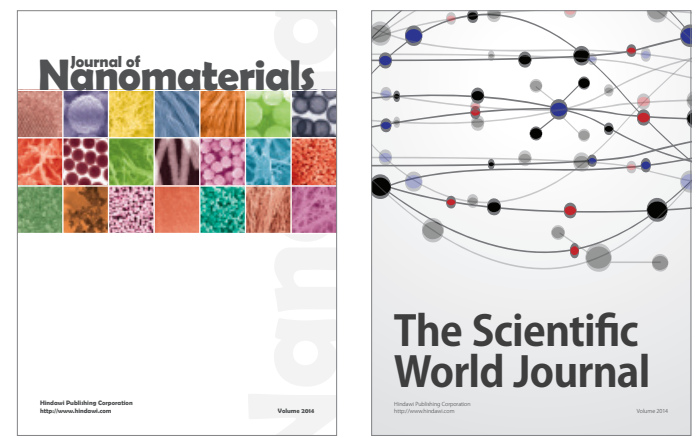

The Scientific World Journal
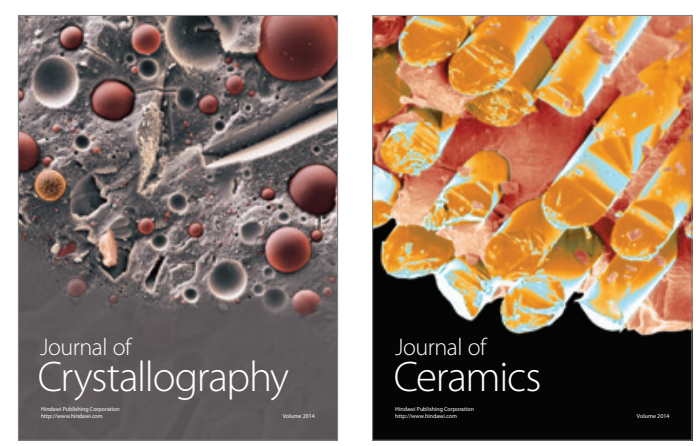
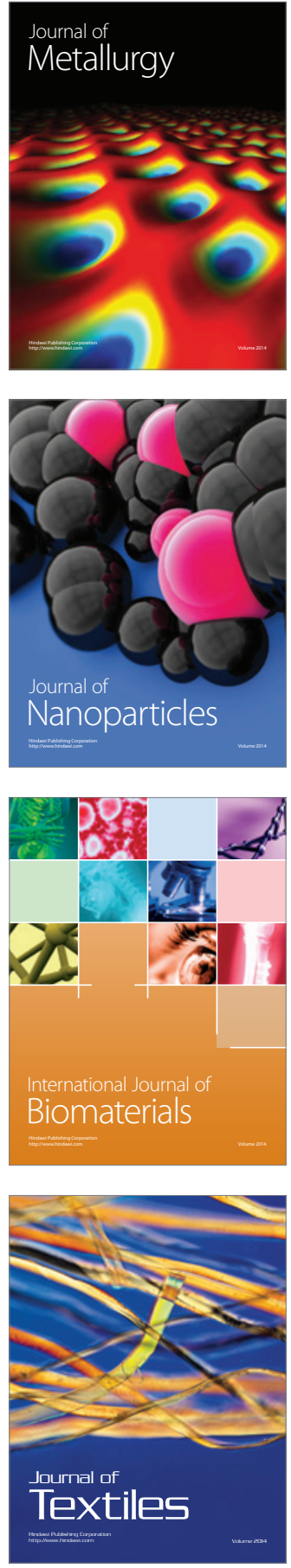